\title{
Heart Rate Variability Time Analyses of the Streptozotocin- Diabetic Rat
}

\author{
M. Jacobson*1, F.C. Howarth², E. Adeghate², K. Fatima-Shad ${ }^{3}$ \\ ${ }^{* 1}$ College of Engineering, UAE University, Al-Ain, P.O. Box 17666, U.A.E. \\ 2 Faculty of Medicine, UAE University, Al-Ain, P.O. Box 17666, U.A.E. \\ 3 Faculty of Science, UAE University, Al-Ain, P.O. Box: 17666, U.A.E.
}

Received 19 September 2005; accepted 10 May 2006

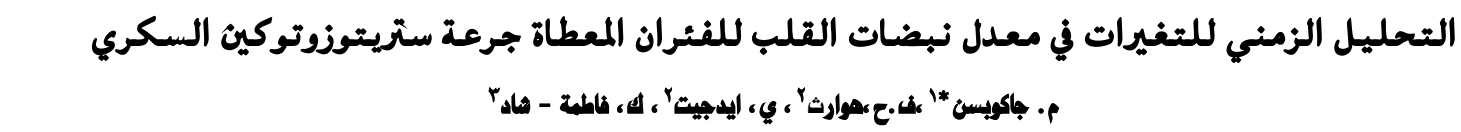

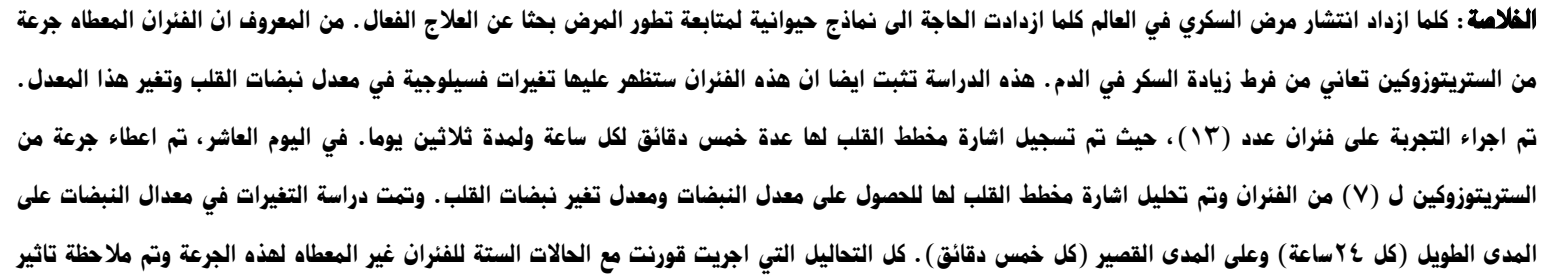

الهنزداه الهنتامية: تغير معدل نبضات القلب، التحليل في مجال الزهن ، الستريتوزوكين ، السكري.

\begin{abstract}
As the world prevalence of diabetes mellitus (DM) increases, animal models of the disease's progression are required for researching effective treatment. The streptozotocin (STZ) treated rat is known to cause hyperglycaemia. This study confirms that this animal model also displays DM physiological effects in the animal heart rate (HR) and heart rate variability (HRV). In particular, 5 minutes of rat $(n=13)$ electrocardiogram (ECG) is acquired hourly for 30 days. At day 10 , the animal $(n=7)$ is dosed with STZ and the ECG is analyzed in order to determine the HR and HRV. The HRV is indexed using two time-based analyses, based on long-term (24hr) and short-term (5min) analyses. All analyses are compared to control non-STZ dosed animals ( $\mathrm{n}=6)$ and display significant DM effects.
\end{abstract}

Keywords: Heart tate variability, Time-domain analysis, Streptozotocin-Diabetic

\section{Introduction}

The world prevalence of DM is projected to increase from 135 million in 1995 to 300 million in 2025 (King, H., Aubert, R.E. and Herman, W.H., 1988). Cardiovascular complications are the most common causes of morbidity and mortality in diabetic patients (Amos, A.F., McCarty, D.J. and Zimmet, P., 1997). Diabetic cardiomyopathy is associated with a variety of myocardial dysfunctions including impairment of contractile force generation, abnormal ventricular filling and delayed relaxation of cardiac muscle and these defects can occur in the absence of vascular complications (Dhalla, N.S., Pierce, G.N., Innes, I.R. and Beamish, R.E., 1985; Howarth, F.C., Qureshi, A., Lawrence, P. and Adeghate, E., 2000; Howarth, F.C., Qureshi, M.A., Bracken, N.K., Winlow, W. and Singh, J., 2001; Howarth, F.C. Qureshi, M.A. and White, E., 2002).

*Corresponding author’s e-mail: mjacob@ieee.org
Early detection of DM, prior to clinical events, coupled with implementation of preventive management strategies, can delay the progression of the disease. In fact, proper diet, physical exercise, cessation of smoking, control of blood pressure, and reduced cholesterol lower a patient's odds of myocardial infarction by 19 times (Wood, D., 1998; Vinik, A. and Flammer, M., 2002).

The HR is controlled by the sympathetic and parasympathetic branches of the autonomic nervous system (ANS). The parasympathetic vagal nerves slow the HR through the release of acetylcholine while the sympathetic nerves accelerate the HR through the release of noradrenaline. The variability of the HR is the result of a balance between the sympathetic and parasympathetic nerves (Bootsma, M., Swenne, C.A., Van Bolhuis, H.H., Chang, P.C., Cats, V.M. and Bruschke, A.V., 1994).

Fluctuations in HR are known to be quasi-periodical, repeating with a period, which is not strictly constant, showing changes in morphology, amplitude and phase from one beat to the next. In steady state conditions, these 
oscillations are maintained around a certain mean value. During periods of rest, the parasympathetic response dominates and the HR lowers. During activity, the sympathetic response dominates and the HR increases (Ori, Z., et al. 1992).

Change in the HR is termed the HRV. Since the HR is controlled primarily by the ANS, assessment of the HRV provides important information about sympathetic and parasympathetic cardiovascular control (Cerutti, S., Bianchi, A.M. and Mainnardi, L.T., 1995). In case of dysfunction, compensatory changes occur in the ANS control system in order to keep the entire circulatory system within normal operating limits. In particular, the presence of DM is known to reduce HRV and disrupt the sympathetic and parasympathetic nervous systems in humans (Bootsma, M., Swenne, C.A., Van Bolhuis, H.H., Chang, P.C., Cats, V.M. and Bruschke, A.V., 1994; Karkuszewski, L., Bissinger, A., Rosaik, M., Kuberska-Kedzierska, M. and Rogulski, B., 2005; Al-Hazimi, A., Al-Ama, N., Syiamic, A., Qosti, R. and Abdul-Galil, K., 2002).

In order to develop clinical screening procedures, appropriate animal models are required to develop the methodology and research possible treatment. The STZ dosed rat displays hyperglycaemia and this study confirms DM physiological effects on HRV comparable to humans.

\section{Method}

\subsection{Animal Preparations}

The ECG was monitored in 13 young, age-matched, adult male Wistar rats using a telemetry system (Data Sciences Int., USA). The system was comprised of transmitter devices, associated receivers, a data exchange matrix and personal computer (PC) for system configuration, control, acquisition, and storage (Fig 1). The transmitters were surgically implanted under general anesthesia and inserted into the peritoneal cavity with the electrodes arranged in the Einthoven bipolar - Lead II configuration (right foreleg and left hind leg).

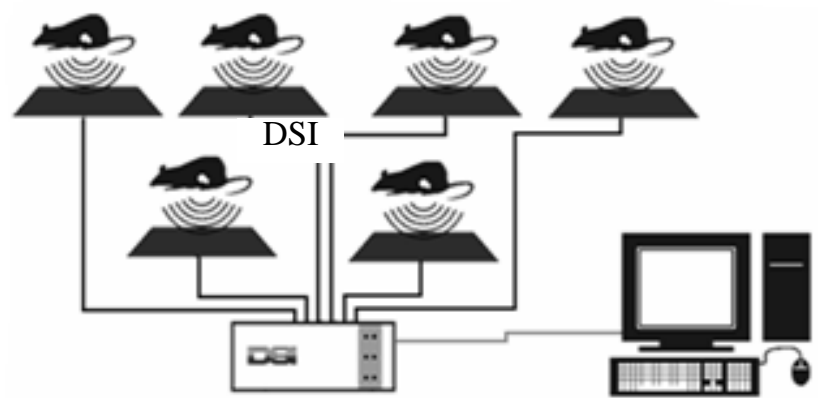

Figure 1. Telemetry system to collect diabetic and control ECG

One week after surgery, transmitters in the animals were activated via magnetic switches and baseline data was recorded for 10 days before induction of DM by administration of the drug streptozotocin in 7 of the 13 rats. Streptozotocin causes selective pancreatic-cell necrosis, which results in hypoinsulinaemia, hyperglycaemia and reduced body weight gain (Howarth, F.C.,
Qureshi, A., Lawrence, P. and Adeghate, E., 2000; Howarth, F.C., Qureshi, M.A., Bracken, N.K., Winlow, W. and Singh, J., 2001; Howarth, F.C. Qureshi, M.A. and White, E., 2002; Choi, K.M., Zhong, Y., Hoit, B.D., Grupp, I.L., Hahn, H., Dilly, K.W., Guatimosin, S., Lederer, W.J. and Matlib, M.A., 2002; Okayama, H., Hamada, M. and Hiwada, K., 1994; Ren, J. and Davidoff, A.J., 1997). Ethical approval for the project was obtained from the Faculty of Medicine \& Health Sciences Ethics Committee for Animal Research of the United Arab Emirates University.

\subsection{Physiological Data Acquisition}

Per the guidelines of the European Society of Cardiology and the North American Society of Pacing and Electrophysiology (Task Force of European Society of Cardiology and North American Society of Pacing and Electrophysiology, 1996), the ECG was sampled at $1 \mathrm{kHz}$, for 5 minutes each hour over one month. Since the average rat HR is about 300 beats per minute (BPM), a 5minute sample ensures measurement of approximately 1500 contractions.

From the ECG signal, the R-waves of the QRS complexes were determined using a secant detector and the beat-to-beat HR was computed. Ectopic beats, such as pre-ventricular contractions (PVC) and skipped beats, were visually removed. The resulting signal was then used for computation of the 5 minute average HR and HRV analyses.

\subsection{HRV Analysis}

The 24-hour, long-term HRV analysis was computed as the standard deviation of the mean 5-minute normalto-normal HR, which is termed the SDANN per (1), where $\overline{\mathrm{HR}}$ is the 5 -minute average heart rate and $\overline{\mathrm{HR}}_{24}$ is the 24-hour average heart rate (Kleiger, R.E., Stein, P.K., Bosner, M.S. and Jeffrey, N. R., 1995). This analysis highlights changes in the HR range o ver one circadian cycle of 24 hours.

$$
\mathrm{SDANN}=\sqrt{\frac{1}{24} \sum_{\mathrm{i}=1}^{24} \overline{\mathrm{HR}}_{\mathrm{i}}^{2}-\overline{\mathrm{HR}}_{24}^{2}}
$$

Specifically, the SDANN is computed by measuring the ECG and determining the 5-minute average HR each hour for 24 hours. Then, the standard deviation of 24 successive samples is determined. Thus, the measurement is spans circadian cycle and indexes the range of HR over maximum and minimum activity.

The short-term HRV analysis determined only from the 5-minute ECG. Specifically, the standard deviation of the 5-minute, normal-to-normal (SDNN) HR is computer per (2), where HRi is the beat-to-beat HR, $\mathrm{N}$ is the number of beats, and is the 5-minute average heart rate (Kleiger, R.E., Stein, P.K., Bosner, M.S. and Jeffrey, N.R., 1995).

$$
\mathrm{SDNN}=\sqrt{\frac{1}{\mathrm{~N}} \sum_{\mathrm{i}=1}^{\mathrm{N}} \mathrm{HR}_{\mathrm{i}}^{2}-\overline{\mathrm{HR}}^{2}}
$$


Thus, the SDNN indexes changes in the HR over a 5minute period. The activity and circadian cycle of the animal are not considered. Thus, though both the SDANN and SDNN both give measurements of the HRV, one indexes 24-hourr HR changes while the other focuses on beat-to-beat changes. In other words, though both are derived from the ECG, each measures a different type of HRV: 24-hour long-term and 5-minute short-term HRV.

\section{Results}

The blood glucose of the 13 rats was measured at days 3 and 20, per Fig. 2. The STZ-treated rats displayed elevated blood glucose confirming the presence of DM while the control rats displayed normal blood glucose levels.

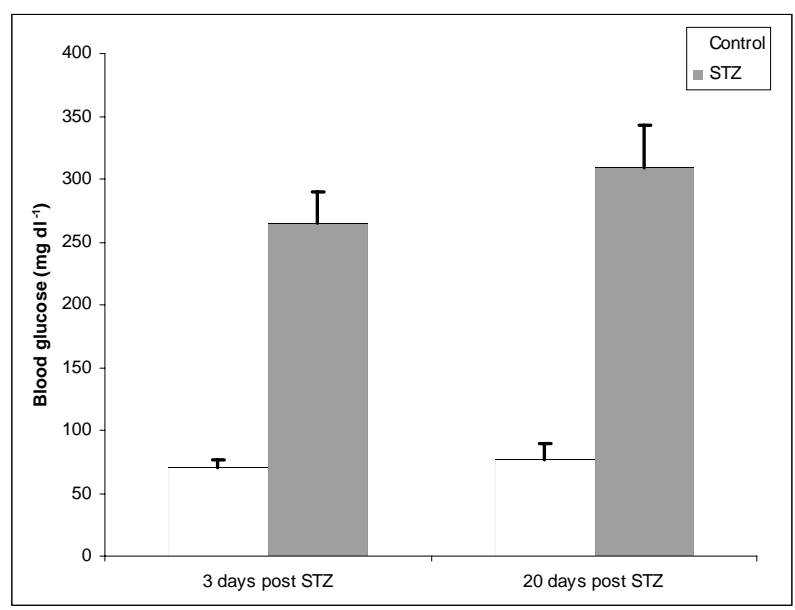

Figure 2. STZ and control group blood glucose levels

The 5-minute average HR is plotted in Fig. 3 for the STZ and control groups. STZ treatment occurred on day 0 and the STZ group $(n=6)$ displayed a drop in HR from day 0 to day 5 , until a new steady-state HR is achieved. At day 20, there is an approximate 80BPM significant difference between the STZ and control groups. Significant testing is accomplished utilizing the t-test with $\mathrm{p}<0.001$ (considered significant).

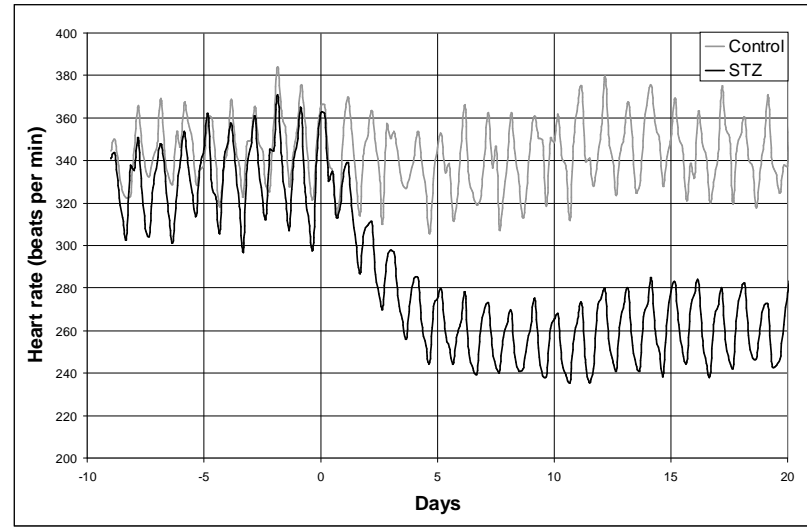

Figure 3. Comparison in average 5 minute HR between STZ and control groups

The standard deviation of 24 successive, 5-minute HR averages is used to determine the SDANN, per the previ- ous discussion. Figure 4 displays the results of the STZ and control groups. After treatment, the STZ group SDANN results are immediately unclear but are obviously declining at day 5 , while the control group SDANN increased. The difference between the groups continues increasing and is significant at day 20.

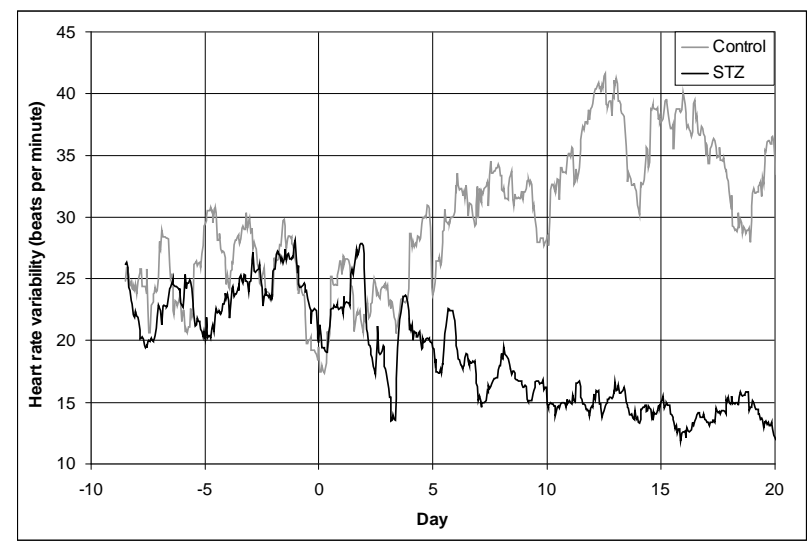

Figure 4. Comparison in HRV SDANN between STZ and control groups

Per the previous discussion, the standard deviation of the 5-minute, normal-to-normal beats in the ECG is used to computed the SDNN. This short-term HRV analysis is displayed in Fig. 5 for the STZ and control groups. After treatment, the SDNN of the STZ group immediately became decreasing to a new steady state value at about day 8 . This new value is about $10 \mathrm{BPM}$ less than pre-STZ treatment. The control group SDNN values, though varied, continue much the same throughout the 30 day experiment.

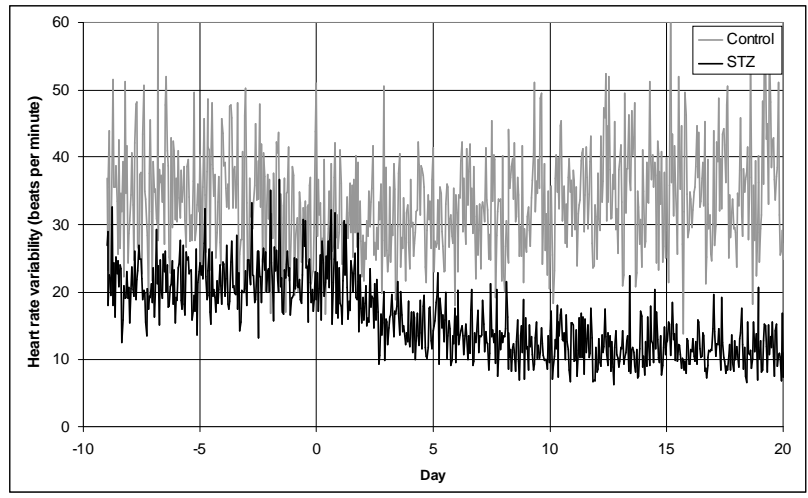

Figure 5. Comparison in SDNN HRV between STZ and control groups

In order to better compare between the two time-based HRV algorithms, a 24-hour moving average is applied to the SDNN. Therefore, the time basis of each HRV index spans 24 hours. Furthermore, in order to compare between the relative changes due to STZ treatment, each is normalized and plotted in Fig. 6. Observably, each HRV index gives a similar deflection due to STZ treatment. The specific amplitude changes over the 30-day experiment are recorded in Table 1 along with the associated standard error. Note that each HRV measurement gives a similar normalized magnitude and error over the experiment period. Statistically, the SDANN and SDNN have a 0.96 cor- 
relation coefficient indicating that the two HRV measurements are highly correlated and give a similar index in response to STZ treatment.

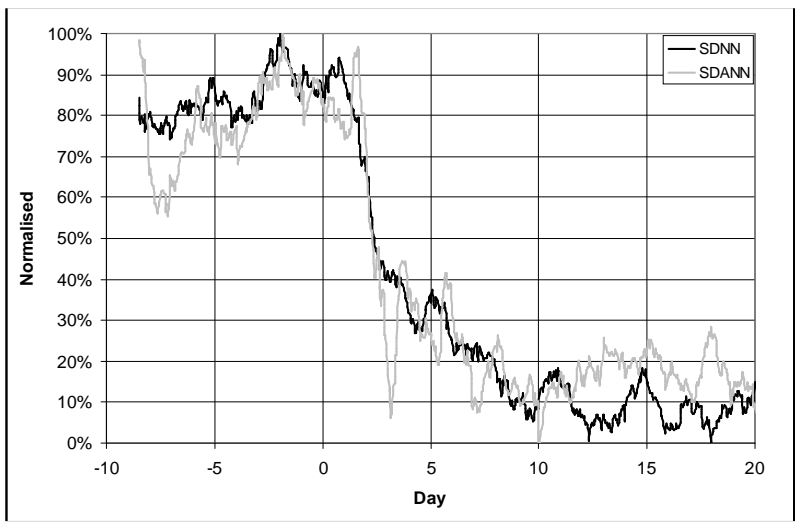

Figure 6. Comparison between the 24-hour average SDNN and SDANN for the STZ group

Table 1. Normalized 24-hour average values and error for the SDNN and SDANN HRV indices

$\begin{array}{ccc}\text { Day } & \text { SDNN } & \text { SDANN } \\ -5 & 85 \pm 2 \% & 76 \pm 3 \% \\ 0 & 87 \pm 2 \% & 85 \pm 3 \% \\ 5 & 33 \pm 3 \% & 25 \pm 3 \% \\ 10 & 11 \pm 4 \% & 10 \pm 5 \% \\ 15 & 14 \pm 2 \% & 22 \pm 2 \% \\ 20 & 11 \pm 2 \% & 9 \pm 5 \%\end{array}$

\section{Discussion}

The SDNN and SDANN analyses of the HRV both display similar changes in response to the STZ treatment. Specifically, after STZ treatment, the HRV begins decreasing to a new steady-state value over a 10 day period. Reduction in HRV is indicative of autonomic neuropathy, specifically the control of the HR. Autonomic neuropathy in known to occur in the presence of $\mathrm{DM}$, and so measurement of the HRV may be used in the screening of DM. SDANN and SDNN are two types of time-based HRV analyses. Though the SDANN is based on long-term (24 hours) measurement and the SDNN is based on shortterm (5-minutes) measurement, each analysis, when normalized and adjusted to the same time scale is found to give a similar HRV deflection in the presence of DM with a similar measurement error. Considering this result in clinical setting, it is likely that short-term HRV measurement, utilizing the SDNN, is sufficient for the screening of DM.

\section{Conclusions}

The SDNN and SDANN analyses of the HRV both display changes in the animal's physiology due to STZ administration, and thus, DM, as has been shown in humans. Both HRV indices are highly correlated and both display a similar reduction in the HRV in comparison to control groups, thus confirming the DM physiological effects of the STZ treated rat model.

\section{Acknowledgments}

This project was supported by Interdisciplinary Grant (01-05-8-12/03) from the United Arab Emirates University.

\section{References}

Al-Hazimi, A., Al-Ama, N., Syiamic, A., Qosti, R. and Abdel-Galil, K., 2002, "Time-domain Analysis of Heart Tate Variability in Diabetic Patients with and without Autonomic Neuropathy, Annals of Saudi Medicine," Vol. 22, pp. 5-6.

Amos, A.F., McCarty, D.J. and Zimmet, P., 1997, "The Rising Global Burden of Diabetes and its Complications: Estimates and Projections to the Year 2010,” Diabetic Medicine, 14: S1-S85.

Bootsma, M., Swenne, C. A., Van Bolhuis, H. H., Chang, P. C., Cats, V. M. and Bruschke, A. V., 1994, "Heart Rate and Heart Rate Variability as Indexes of Sympathovagal Balance,” Am. J. Physiol 266, H1565-H1571.

Cerutti, S., Bianchi, A. M. and Mainardi, L. T., 1995, "Spectral Analysis of the Heart Rate Variability Signal,” Heart Rate Variability, Futura Publishing Company.

Choi, K.M., Zhong, Y., Hoit, B.D., Grupp, I.L., Hahn, H., Dilly, K.W., Guatimosin, S., Lederer, W.J. and Matlib, M.A., 2002, "Defective Intracellular Ca2+ Signaling contributes to Cardiomyopathy in Type 1 Diabetic Rats,” Am J Physiol, 283: H1398-H1408.

Dhalla, N.S., Pierce, G.N., Innes, I.R. and Beamish, R.E., 1985, "Pathogenesis of Cardiac Dysfunction in Diabetes Mellitus,” Can J Cardiol, Vol. 1, pp. 263281.

Howarth, F.C., Qureshi A, Lawrence P. and Adeghate E., 2000, "Chronic Effects of Streptozotocin-induced Diabetes on the Ultrastructure of Rat Ventricular and Papillary Muscle,” Acta Diabetol, Vol. 37, pp. 119124.

Howarth, F.C., Qureshi, M.A. and White, E., 2002, "Effects of Hyperosmotic Shrinking on Ventricular Myocyte Contraction and Intracellular $\mathrm{Ca} 2+$ in Streptozotocin-induced Diabetic Rats,” Pflügers Archiv, Vol. 444, pp. 446-451.

Howarth, F.C., Qureshi, M.A., Bracken, N.K., Winlow, W. and Singh, J., 2001, "Time-dependent Effects of Streptozotocin-induced Diabetes on Contraction of Ventricular Myocytes from Rat Heart," Emirates Medical Journal, Vol. 19, pp. 35-41.

King, H., Aubert, R.E. and Herman, W.H., 1998, “Global Burden of Diabetes, 1995-2025: Prevalence, Numerical Estimates, and Projections,” Diabetes Care, Vol. 21, pp. 1414-1431. 
Kleiger, R. E., Stein, P. K., Bosner, M. S. and Jeffrey, N. R., 1995, "Timed-doman Measurements of Heart Rate Variability,” Heart Rate Variability, Futura Publishing Company.

Markuszewski, L., Bissinger, A., Rosiak, M., KuberskaKedzierska, M. and Rogulski, B., 2005, "Heart Rate Turbulence and Heart Rate Variability in Patients with Diabetes Mellitus,” Folia Cardiologica, 12, supl. C; p. 181.

Okayama, H., Hamada, M. and Hiwada, K., 1994, "Contractile Dysfunction in the Diabetic-Rat Heart is an Intrinsic Abnormality of the Cardiac Myocyte," Clinical Science, Vol. 86, pp. 257-262.

Ori, Z., et al. 1992, "Heart Rate Variability," Frequency Domain Analysis, Cardiology Clinics, Vol. 10(3), pp. 499-537.

Ren, J. and Davidoff, A.J., 1997. "Diabetes Rapidly
Induces Contractile Dysfunctions in Isolated Ventricular Myocytes,” Am J Physiol, Vol. 272, pp. H148-H158.

Task Force of the European Society of Cardiology and the North American Society of Pacing and Electrophysiology., 1996, "Heart Rate Variability, Standards of Measurement, Physiological Interpretations, and Clinical Use," European Heart Journal, Vol. 17, p. 354.

Vinik, A. and Flemmer, M., 2002, "Diabetes and Macrovascular Disease," J. of Diabetes and its Complications, Vol. 16, pp. 235-245.

Wood, D., 1998, "Prevention of Coronary Heart Disease in Clinical Practice," Recommendations of the Second Joint Task Force of European and other Societies on Coronary Prevention, Arteriosclerosis, Vol. 140, pp. 199-270. 